\title{
Systematic review on the incidence of low back pain as well as on the rehabilitation treatment methods used
}

\section{IONITE Catalin ${ }^{1}$, ROTARIU Mariana ${ }^{1}$, CONDURACHE Iustina ${ }^{1}$}

Corresponding author: ROTARIU Mariana, E-mail: rotariu29@yahoo.com,

1. Faculty of Medical Bioengineering, "University of Medicine and Pharmacy Grigore T Popa”, Iasi, Romania

\begin{abstract}
Introduction. Low back pain is a growing condition both in terms of sampling and incidence, reaching to affect any of us at some point. Material and method. In order to write the article, four international libraries were used, where search criteria were used such as: "low back pain", "low back pain incidence" and "low back pain treatment". The articles used in this paper were carefully selected according to their relevance for this study, but only considered articles published in 2020. Results and discussions. The obtained results indicated that both physical therapy and physiotherapy have beneficial effects in ameliorating the symptoms generated by low back pain, but due to the lack of an optimal protocol patients turn to alternative therapies that have shown greater efficacy than physical therapy in amelioration. symptoms generated by low back pain. Conclusions. The lack of an optimal physiotherapeutic treatment protocol causes patients to turn to alternative therapies that are more effective, as well as a lower cost per treatment.
\end{abstract}

Keywords: low back pain, incidence, prevalence, protocol, treatment,

\section{Introduction}

Low back pain is a condition that can be found in both the acute and chronic stage. Low back pain will affect almost any of us at some point in life.

Causes that can lead to low back pain can be: congenital (skeletal irregularities, spina bifida), injuries (sprains and traumatic injury), degenerative problems (intervertebral disc degeneration, spondyloss, arthritis, other inflammatory disease), nerve and spinal cord problems ( spinal nerve compression, sciatica, spinal stenosis, spondylolisthesis, herniated or ruptured disc, infections, cauda equine syndrome, osteoporosis), non-spine sourches (kidney stones, endometriosis, fibromyalgia, tumors and pregnancy).

The prevalence and incidence of this disease is different depending on the type of activity practiced, but even so the incidence is increasing.

In 2019, Karel K. conducted a systematic review containing data from 7 countries such as Canada, the United States, Sweden, Belgium, Finland, Israel and The Netherland, finding that the lower back pain levy had a wide range from $1.4 \%-20 \%$, with an incidence between $0.02 \%-7.0 \%$ (1). This information was also confirmed by Francis F. also in 2019 (2). The data were compared by Karel K. with data obtained by Germany, where the prevalence was $3.7 \%$ and an incidence of $1.3 \%$ for a first diagnosis of low back pain (1). A study by Hülya Y. at Medicine at Bezmialem Vakıf University in 2016, wanted to show the incidence of low back pain among university students, showing that the female group had an incidence of $91.3 \%$ (355 out of 471 female participants) suffered / suffers from low back pain in recent years with the following classification: $174(33.46 \%)$ were from the
School of Dentistry, $110(21.15 \%)$ were from the Faculty of Pharmacy, $153(29.42 \%)$ were from the Faculty of Health Sciences, and $83(15.96 \%)$ were not from the School of Medicine (3). Not only medical university students were used in the study to identify the incidence of low back pain, Kanchanomai S., conducted in 2015 at a public university in Thailand a study on a batch of 684 patients, identifying an incident $31 \%$ in low back pain (4). In addition to identifying the incidence of low back pain among young people, Chaiklieng $\mathrm{S}$. tried to find out the incidence of this condition on the administrative staff of the University of Thailand (159 workers), showing that the incidence of low back pain over a period of 12 months was $83 \%$ (5). This condition makes its presence felt among athletes where low back pain can be identified in weightlifters, where Shimozaki K., conducted in 2018 a study for a period of 3 years, 12 subjects (weightlifters), and showed that in the second year of study 1 participant out of 12 was diagnosed with low back pain, and at the end of the 3 years, 312 participants was diagnosed with low back pain, meaning an incidence of $25 \%$ within 3 years (6). Running is not excluded by this problem, Maselli F., showed in 2020 that it has a low values prevalence $(0.7-20.2 \%)$ and incidence $(0.3-22 \%)$ of low back pain among runners (7). Neither rowers are left out of this pathology, Newlands C. in 2015, showing that the incidence in this sport is $6 \%-25 \%$ during a year (8).

This shows us how low back pain affects the entire population regardless of: the type of physical activity practiced, the type of professional activity, age, sex, race, etc. 


\section{Material and method}

In order to write this paper, four international libraries were searched in the international database, such as: Thomson ISI - Web of Science, Scopus, Springer Link, Science Direct. The search criteria used were "low back pain", "low back pain incidence" and "low back pain treatment". The articles used in this paper were carefully selected according to their relevance for this study, but they considered only the articles published in 2020 .

In order to complete this paper, the aim was to identify a prevalence of low back pain and its incidence both among normal people and among performance and leisure athletes. In addition to those mentioned, the types of treatment were followed in the literature, as well as the protocols used in treating the condition or ameliorating the symptoms generated by low back pain.

\section{Results and discussions}

The literature provides us with information about the results obtained from the use of physical therapy, but does not expose the protocols used in conducting studies. Walston Z. demonstrated the importance of early improvement in the treatment of low back pain with physical therapy in a group that included 11,945 patients, concluding the importance of the rapid start of physical therapy as well as roughly two-thirds of their improvement in the first $40 \%$ of visits regardless of their symptom duration (9). The same thing was done by Vadala G. conducting a review where tried to identify the role of physical activity in the treatment of low back pain. It is highlighted that physical activity reduces the patient's pain and improves his motor skills $(10,11)$. Therapists are constantly trying to find new ways to treat and promote low back pain. Grande-Alonso M. combined therapeutic exercise with therapeutic education in 2020 , leading to a reduction in pain intensity, a reduction in disability and an increase in lumbar strength (12).

Other therapists have tried to address low back pain only through physiotherapy, thus demonstrating its effectiveness (13).

Batistella C.E. He resorted to physiotherapy in the low back pain approach, using Russian current for a period of 4 weeks, demonstrating its effectiveness in reducing the pain felt, as well as short-term improvements in resistance and thickness changes of the multifidus (14).

Ozkaraoglu D.K. resorted to physiotherapy, comparing the efficacy of High Intensity Laser Therapy (HILT) and Transcutaneous Electrical Nerve Stimulation (TENS) in low back pain in a group of 40 patients, demonstrating that High Intensity Laser Therapy is more effective than TENS in terms of pain reduction. (15).

Lacking an optimal therapeutic treatment protocol, patients are turning to alternative methods of treatment. Khodakarami N. made a cost comparison between Physical Therapy and Chiropractic manipulations in low back pain cases, concluding that the cost for patients included in the chiropractic manipulation group was $\$$ 48.56 lower than that of the Physical group. therapy. Which pushes us to find the optimal treatment in terms of physical therapy, which will increase efficiency and lower treatment costs (16). Meirelles F.D. compared the effects of osteopathic manipulation techniques with the effects of therapeutic exercises in cases of chronic nonspecific low back pain and showed that both methods had positive effects in reducing the symptoms generated by low back pain, only that the effects produced by osteopathic manipulations have generated much better results than the effects generated by therapeutic exercises (17). Yu S.Y. used acupuncture as a treatment for low back pain, thereby reducing the pain felt by the patient (18). Majid S. shows us in a review of the literature, conducted in 2020, that the effects of yoga practice has the potential to reduce pain and improve the quality of life in cases of low back pain (19). Cheon C. wanted to show in 2020 the most common methods of medical treatment used by the Korean medical system in the treatment of low back pain, mentioning that the most used au fost: acupuncture alone, a combination of acupuncture, mox- ibustion, cupping, and physiotherapy, and a combination of acupuncture and physiotherapy (20).

At the same time, those in the pharmacological field are constantly trying to find new things that will improve the patient's quality of life. Yang S. succeeded in 2020 in reducing the pain felt by the patient and improving the patient's functions with chronic low back pain through nerve growth factor $(21,22)$.

\section{Conclusion}

Low back pain is a condition that will affect almost any of us at some point.

The literature shows us a lack of an optimal treatment protocol in the treatment of low back pain. Finding a protocol is essential to reduce the recovery time and thus the total cost of treatment.

The lack of a treatment protocol pushes patients to different alternative treatment methods that are currently demonstrating superior physical therapy efficacy. The literature has not shown any study to use a medical dynamic orthosis after treatment to maintain the results obtained after physical therapy treatments. Maintaining results with medical orthoses can increase the durability of treatment and reduce costs, which could help the patient both socially and economically.

\section{Author contributions.}

All the authors had the same contribution.

\section{Acknowledgements.}

This article belongs to the project ESTABLISHMENT OF RESEARCH DEPARTMENT INNOVATIVE METHODS OF TREATMENT OF NEUROLOCOMOTOR SYSTEM DISEASES, Priority Axis no.1, 111-POC, SMIS- 121822. 


\section{References:}

1. Karel K. Real-world prevalence and incidence of lowe back pain in Germany. Rheumatology International. 2019; 39(6): 1113-1113. https://linkspringer-

com.dbproxy.umfiasi.ro/article/10.1007/s00296019-04295-8

2. Francis F., Tadesse G., Isaac O. Real-world incidence and prevalence of low back pain using routinely collected data. Rheumatology International. 2019;39(4): 619-626. https://linkspringer-

com.dbproxy.umfiasi.ro/article/10.1007/s00296019-04273-0

3. Hülya Y. Perihan T.. Incidence and Risk Factors of Low Back Pain in Students Studying at a Health University. Bezmialem Science. 2016; 40(1): 12-18. http://cms.galenos.com.tr/Uploads/Article_20355/B AS-4-12-En.pdf

4. Kanchanomai S., Janwantanakul P., Pensri P. Jiamjarasrangsi W.. A Prospective Study of Incidence and Risk Factors for the Onset and Persistence of Low Back Pain in Thai University Students. Asian-Pacific Journal of Public Health. 2015; $27 \quad$ (2): $\quad$ NP106-NP115 http://apps.webofknowledge.com.dbproxy.umfiasi.ro /full_record.do?product $=$ WOS\&search_mode $=$ Gene ralSearch\&qid $=3 \& S I D=C 4 j B h z s w M m V K S b x 7 F w P$ \&page $=1 \&$ doc $=25 \&$ cacheurlFromRightClick $=$ no

5. Chaiklieng S., Suggaravetsiri P., Stewart J.. Incidence and risk factors associated with lower back pain among university office workers. International Journal of Occupational Safety and Ergonomics. DOI: 10.1080/10803548.2019.1706827 International Journal of Occupational Safety and Ergonomics

http://apps.webofknowledge.com.dbproxy.umfiasi.ro /full_record.do?product=WOS\&search_mode $=$ Gene ralSearch\&qid $=3 \& S I D=C 4 j B h z s w M m V K S b x 7 F w P$ \&page $=1 \&$ doc $=4 \&$ cacheurlFromRightClick $=$ no

6. Shimozak K., Nakase J., Yoshioka K,. Takata Y., Asai K., Kitaoka K., Tsuchiya H.. Incidence rates and characteristics of abnormal lumbar findings and low back pain in child and adolescent weightlifter: A prospective three-year cohort study. Plos One. 2018; 13(10): Article Number: e0206125 https://journals.plos.org/plosone/article?id=10.1371/j ournal.pone. 0206125

7. Maselli F., Storari L., Barbari V., Colombi A., Turolla A., Gianola S., Rossettini G., Testa M.. Prevalence and incidence of low back pain among runners: a systematic review. BMC Musculoskeletal
Disorders. 2020; 21(1): 343, DOI: 10.1186/s12891020-03357-4 https://link-springercom.dbproxy.umfiasi.ro/content/pdf/10.1186/s12891 -020-03357-4.pdf

8. Newlands C., Reid D., Parmar P.. The prevalence, incidence and severity of low back pain among international-level rowers. British Journal of Sports Medicine. $\quad 2015 ; \quad 49(14)$ : $951-956$ https://bjsm.bmj.com/content/49/14/951.short

9. Walston Z .,McLester C.. Importance of Early Improvement in the Treatment of Low Back Pain With Physical Therapy. Spine. 2020; 45 (8): 534540

https://journals.lww.com/spinejournal/Abstract/2020 /04150/Importance_of_Early_Improvement_in_the Treatment.10.aspx? context $=$ FeaturedArticles\&collec tionId $=1$

10. Vadala G., Russo F., De Salvatore S., Cortina G., Albo E.,Papalia R., Denaro V .. Physical Activity for the Treatment of Chronic Low Back Pain in Elderly Patients: A Systematic Review. Journal of Clinical Medicine. 2020; 9 (4): Article number: 1023

https://www.mdpi.com/2077-

0383/9/4/1023/htm?fbclid=IwAR2HJuukHknp2q8de nBp_CSvnoMVezaDwbQrI4XL5op-

4BJ5NHpJVtXHiek

11. Rotariu M., Ionite C., Turnea M. Modeling and simulation of statistic demands of the vertebral column in the sagittal plane. The 14th International Scientific Conference eLearning and Software for Education Bucharest, Vol. 3. Pag. 475-480, 2018 http://proceedings.elseconference.eu/index.php?r=sit e/index\&year $=2018 \&$ index $=$ papers\&vol=29\&paper $=13 \mathrm{c} 0 \mathrm{ea} 4 \mathrm{a} 937 \mathrm{a} 9277834036 \mathrm{~d} 818 \mathrm{c} 9347 \mathrm{c}$

12. Grande-Alonso M ., Garrigos-Pedron M ., CuencaMartinez F., Vidal-Quevedo C ., Prieto-Aldana M., La Touche R., Gil-Martinez A. Influence of the Generation of Motor Mental Images on Physiotherapy Treatment in Patients with Chronic Low Back Pain. Pain Physician. 2020;24(4):E3990$+$

https://www.painphysicianjournal.com/current/pdf?a rticle $=$ NzA5MA $\% 3 \mathrm{D} \% 3 \mathrm{D} \&$ journal $=128$

13. Rotariu M., Turnea M., Ionite C.. A different approach to the use of elastic bands in the recovery of lower lumbar discopathy. Balneo Research $\begin{array}{lllll}\text { Journal. } & 2019 ; & 10 & \text { (3): } \quad 213-217\end{array}$ http://bioclima.ro/Balneo259.pdf

14. Batistella C.E., Bidin F., Giacomelli I., Nunez M.A., Gasoto E., de Albuquerque C.E.,Flores L.J.F., Bertolini G.R.F., Effects of the Russian 
current in the treatment of low back pain in women: A randomized clinical trial., Journal of Bodywork and Movement Therapies. 2020; 24 (2): 118-122

http://apps.webofknowledge.com.dbproxy.umfiasi.ro /full_record.do?product $=$ WOS\&search_mode $=$ Gene ralSearch\&qid=1\&SID=D2eI8VFWZLLyWjZtLgZ \&page $=1 \&$ doc $=38 \&$ cacheurlFromRightClick $=$ no

15. Ozkaraoglu D.K., Tarakci D., Algun Z.C. Comparison of two different electrotherapy methods in low back pain treatment. Journal of back and musculoskeletal rehabilitation. 2020; 33 (2): 193199

http://apps.webofknowledge.com.dbproxy.umfiasi.ro /full_record.do?product=WOS\&search_mode $=$ Gene ralSearch\&qid=1\&SID=D2eI8VFWZLLyWjZtLgZ \&page $=2 \&$ doc $=62 \&$ cacheurlFromRightClick $=$ no

16. Khodakarami N. Treatment of Patients with Low Back Pain: A Comparison of Physical Therapy and Chiropractic Manipulation. Healthcare. 2020; 8 (1): Article number: 44 https://www.mdpi.com/22279032/8/1/44

17. Meirelles F.D., Cunha J.C.D.M., da Silva E.B. Osteopathic manipulation treatment versus therapeutic exercises in patients with chronic nonspecific low back pain: A randomized, controlled and double-blind study. Journal of back and musculoskeletal rehabilitation. 2020; 33 (3): 367377

http://apps.webofknowledge.com.dbproxy.umfiasi.ro /full_record.do?product=WOS\&search_mode $=$ Gene ralSearch\&qid $=1 \& S I D=D 2 e I 8 V F W Z L L y W j Z t L g Z$ \&page $=2 \&$ doc $=59 \&$ cacheurlFromRightClick $=$ no.

18. Yu S.Y., Ortiz A., Gollub R.L., Wilson G., Gerber J., Park J.,Huang Y.T., Shen W., Chan S.T., Wasan A.D., Edwards R.R., Napadow V., Kaptchuk T.J., Rosen B., Kong J.. Acupuncture Treatment Modulates the Connectivity of Key Regions of the Descending Pain Modulation and
Reward Systems in Patients with Chronic Low Back Pain. Journal of Clinical Medicine. 2020; 9(6): Article number: 1719

https://www.mdpi.com/2077-0383/9/6/1719

19. Majid S., Syahrul S. Effect of yoga intervention among patients undergoing low back pain treatment: A literature review.Enfermerica Clinica. 2020; 30: 177-181

https://www-sciencedirect-

com.dbproxy.umfiasi.ro/science/article/abs/pii/S1 13 0862119304231

20. Cheon C. Traditional Korean medicine treatment patterns in patients with low back pain: A crosssectional study based on the 2017 Korean Medicine Utilization and Herbal Medicine Consumption Survey. European Journal of Integrative Medicine. 2020; 36: Article number 101124

http://apps.webofknowledge.com.dbproxy.umfiasi.ro /full_record.do?product $=$ WOS\&search_mode $=$ Gene ralSearch\&qid $=1 \&$ SID= D2eI8VFWZLLyWjZtLgZ \&page $=1 \&$ doc $=24 \&$ cacheurlFromRightClick $=$ no

21. Yang S ., Huang., Ye Z.Q., Li L., Zhang Y.. The Efficacy of Nerve Growth Factor Antibody for the Treatment of Osteoarthritis Pain and Chronic LowBack Pain: A Meta-Analysis. Frontiers in Pharmacology. 2020;11: Article number: 817 https://www-ncbi-nlm-nihgov.dbproxy.umfiasi.ro/pmc/articles/PMC7339523/

22. Raţă Bogdan C. , Rață Gloria, Rață Marinela, Correlation between the physical growth indices and the motor capacity indicesin lower secondary students, University Annals, Series Physical Education and Sport / Science, Movement and Health, ISSN: 2285-777X, ISSN - L 2285-777X, 2019, Volum: XIX, Nr.: 2 supplement, pp:328- 338 